\title{
Coding for the Initiation of Pseudopregnancy by Temporally Patterned Activation of Amygdalar NMDA Receptors
}

\author{
Michael L. Lehmann, Heather McKellar, and Mary S. Erskine \\ Department of Biology, Boston University, Boston, Massachusetts 02215
}

Female rats modulate the number and interval between the intromissions the female receives during mating. This patterned vaginocervical stimulation (VCS) is critical for triggering long-term changes in prolactin (PRL) secretion necessary for pregnancy or pseudopregnancy (P/PSP). Previous work has shown that NMDA receptor activation in the posterodorsal medial amygdala (MEApd) is required at the time of mating for VCS to induce the twice-daily PRL surges characteristic of P/PSP. The current studies examined whether patterned activation of glutamate receptors within the MEApd induces P/PSP. In anesthetized, cycling females, three $0.27 \mu \mathrm{g}$ NMDA infusions given at $30 \mathrm{~min}$ intervals into the MEApd initiated P/PSP, whereas a single NMDA infusion of the same total dose $(0.8 \mu \mathrm{g})$ had no effect. In conscious, freely behaving females, three infusions of an excitatory amino acid (EAA) mixture applied at the same interval were more effective in initiating P/PSP and nocturnal PRL surges than were single infusions at the same or higher concentrations. Infusion intervals of 5 and $60 \mathrm{~min}$ as well as continuous $1 \mathrm{~h}$ infusion did not induce P/PSP. Finally, a synergistic effect was observed between EAA and mating stimulation, because a subthreshold EAA infusion combined with subthreshold numbers of intromissions induced P/PSP. These results demonstrate that repeated, properly spaced, temporally discreet periods of glutamate receptor activation within the MEApd, which mimic mating stimulation, encode for P/PSP. Such findings suggest that single intromissions normally release individually subthreshold quanta of glutamate within the MEApd that summate to induce P/PSP.

Key words: glutamate; medial amygdala; vaginocervical stimulation; neuroendocrine memory; sensory transduction; pacing

\section{Introduction}

Rat sexual behavior is useful for studying experience-dependent changes in brain function. For mating to be successful, both males and females must experience repeated and intermittent genitosensory stimuli during mating. In males, such stimuli are necessary for ejaculation, whereas in females, such stimuli are necessary for initiation of pregnancy (Erskine, 1995). In the female, a threshold amount of vaginocervical stimulation (VCS) is necessary to initiate the twice-daily prolactin (PRL) surges that are required for early pregnancy or pseudopregnancy (P/PSP), and these surges are expressed in an all-or-none manner once sufficient stimulation has been received (Smith and Neill, 1976; Smith et al., 1976; Gunnet and Freeman, 1983).

Interestingly, both the number and the temporal patterning of vaginal intromissions that the female receives from the male contribute to attainment of the threshold for P/PSP. The probability of P/PSP increases as a function of the number of intromissions, with 10-15 intromissions inducing a reliably higher incidence of P/PSP than three to seven intromissions (Adler, 1969; Edmonds et al., 1972; Erskine et al., 1989). Furthermore, the temporal spac-

Received May 11, 2005; revised Aug. 11, 2005; accepted Aug. 12, 2005.

This work was supported by a Clare Boothe Luce Professorship from the Henry Luce Foundation and by National Institutes of Health Grants MH64187 and MH01435 to M.S.E. We thank Dr. Eva K. Polston for helpful discussions and Dr. A. F. Parlow of the National Hormone and Peptide Program for providing the reagents for the prolactin RIA.

Correspondence should be addressed to Dr. Mary S. Erskine, Department of Biology, Boston University, 5 Cummington Street, Boston, MA 02215. E-mail: erskine@bu.edu.

DOI:10.1523/JNEUROSCI.1893-05.2005

Copyright $\odot 2005$ Society for Neuroscience $\quad 0270-6474 / 05 / 258696-08 \$ 15.00 / 0$ ing that occurs when the female regulates, or paces, intromissions from males contributes to initiation of P/PSP; fewer intromissions are necessary to induce PRL surges when the interval between intromissions is extended by pacing behavior. Thus, information from sequential intromissions is summated in brain, and a short-term mnemonic or memory of an individual mating event is established that allows accumulation of information about repeated VCS.

Substantial evidence suggests that initial summation of inputs from VCS occurs within the posterodorsal medial amygdala (MEApd) (Rowe and Erskine, 1993; Tetel et al., 1993; Polston et al., 2001). This area is unique compared with other matingresponsive brain areas in that mating-induced c-fos expression is proportionate to the number and rate of intromissions the female receives (Polston and Erskine, 1995; Erskine and Hanrahan, 1997) and is completely blocked by bilateral transection of the pelvic nerve that innervates the uterine cervix and vagina (Rowe and Erskine, 1993). We have shown previously that NMDA receptor activation within the MEApd is required for mating-induced P/PSP (Polston et al., 2001). Application of the selective NMDA receptor antagonist AP-5 to the MEApd 15 min before mating completely prevented P/PSP, whereas the same treatment given 90 min after mating had no effect (Polston et al., 2001). Therefore, prolonged changes in neuroendocrine function are initiated through actions of glutamate within the MEApd.

The present experiments tested the hypothesis that induction of P/PSP by intermittent intromissions occurs in response to 
repeated and temporally discreet periods of glutamate receptor activation within the MEApd. We identified doses of NMDA and excitatory amino acids (EAAs) that, when infused unilaterally into the MEApd, were subthreshold for P/PSP and showed that repeated, spaced infusion of individually subthreshold doses is more effective than single infusions of the total dose. In addition, a subthreshold dose of EAAs summated with subthreshold numbers of intromissions to induce P/PSP. The results suggest that neural coding of individual intromissions for P/PSP occurs by repetitive release of EAAs within the MEApd.

\section{Materials and Methods}

Animals

Experimental animals were female Long-Evans rats (225-275 g) obtained from Charles River Laboratories (Wilmington, MA). Mating stimulation was provided by sexually experienced males of the same strain (300-400 g). Animals were housed singly in wire mesh cages with food and water available ad libitum in a room with a reversed light cycle (lights on 8:00 P.M. to 8:00 A.M.) to facilitate procedures conducted during the dark phase of the cycle; times reported here are nonreversed. Ovarian cyclicity of all females was monitored by daily vaginal lavage throughout the experiment, and only females exhibiting two consecutive estrous cycles of $4-5 \mathrm{~d}$ before experimentation were used. P/PSP was considered to have been induced if $8-13 \mathrm{~d}$ of consecutive diestrus smears were observed after treatment (Gunnet and Freeman, 1983). Where possible, vaginal smears continued after treatment until two complete estrous cycles were observed. All procedures were approved by the Institutional Animal Care and Use Committee at Boston University in accordance with National Institutes of Health guidelines.

\section{Administration of NMDA into the MEApd of anesthetized animals}

Females received one or three infusions of NMDA or PBS into the MEApd on the day of vaginal proestrus. To avert seizure induction by NMDA, NMDA was administered while animals were anesthetized. Females were anesthetized with sodium pentobarbital (Nembutal; $50 \mathrm{mg} /$ kg, i.p.; J. A. Webster, Sterling, MA) and placed into a stereotaxic apparatus (David Kopf Instruments, Tujunga, CA). A hole was drilled unilaterally in the left side of the skull, and a filled $2.0 \mu \mathrm{l}$ Hamilton syringe (model 7001) attached to a Kopf microinjector was lowered into the MEApd [anteroposterior (AP) from bregma, -3.0; mediolateral, +3.5; dorsoventral (DV), -8.1] (Paxinos and Watson, 1986). The bevel of the needle was pointed medially to direct the infusate toward the MEApd from the site of penetration at the lateral edge of the optic tract, as reported previously (Polston et al., 2001). Females received either a single infusion of $140 \mathrm{mM}(8.0 \mu \mathrm{g})$ or $14.0 \mathrm{~mm}(0.8 \mu \mathrm{g})$ or three infusions of $4.60 \mathrm{~mm}(0.27 \mu \mathrm{g}$; total dose, $0.8 \mu \mathrm{g}) \mathrm{L}-\mathrm{NMDA}$ (Sigma, St. Louis, MO) in $0.4 \mu \mathrm{l}$ spaced at $30 \mathrm{~min}$ intervals between 10:30 A.M. and 12:00 P.M. Control females received three PBS infusions spaced $30 \mathrm{~min}$ apart. Each infusion was given over $4 \mathrm{~min}$. For single infusions, syringes were left in place for $4 \mathrm{~min}$ after infusion to allow drug diffusion away from the needle tip. For multiple infusions, syringes were left in place for the duration of the three infusions and were removed $4 \mathrm{~min}$ after the third infusion. After removal of the infusion needle, the hole in the skull was filled with bone wax, and the skin was sutured closed.

\section{Administration of EAAs into the MEApd of conscious animals}

To allow infusion of awake animals, guide cannulas were implanted into cycling females using ketamine $\mathrm{HCl}$ (Ketaset; 95 mg/kg, i.p.; Fort Dodge Labs, Fort Dodge, IA) and xylazine (Rompun; $10 \mathrm{mg} / \mathrm{kg}$; Bayer, Shawnee Mission, KS) anesthesia and standard stereotaxic techniques. Guide cannulas were constructed from wide-bore 23 ga stainless-steel injection needles (Becton Dickinson, Franklin Lakes, NJ), as described previously (Polston et al., 2001). They were directed at the left MEApd using the above coordinates except they were lowered until the ventral tip resided $2.0 \mathrm{~mm}$ dorsal to the target site (DV, $-6.1 \mathrm{~mm}$ from dura). They were affixed to the skull with jeweler screws and dental cement (Henry Schein Dental, Melville, NY).

After two estrous cycles occurred postoperatively, a mixture of L-aspartic acid monosodium (Asp; Spectrum, Gardena, CA) and L-glutamic acid monosodium (Glu; Sigma) in a 2:1 ratio with $1 \mu \mathrm{M}$ of the NMDA receptor coagonist glycine (Gly; Aldrich, Milwaukee, WI) was infused into the MEApd at several concentrations (see below) in single or triple infusions between 10:30 A.M. and 12:00 P.M. on vaginal proestrus. Infusion treatments were administered into the MEApd using a beveled 30 ga infusion needle that extended $2.0 \mathrm{~mm}$ beyond the ventral tip of the guide cannula (Coopersmith et al., 1996). The infusion needle was connected by polyethylene tubing (PE20; Clay Adams, Parsippany, NJ) to a 1 $\mu l$ syringe. Once the needle was inserted into the guide cannula, the animal was placed back into her homecage with the cage slightly open to reduce handling stress. Infusates $(0.4 \mu \mathrm{l})$ were delivered at a rate of 0.1 $\mu \mathrm{l} / \mathrm{min}$. Four minutes after infusion, the needle was withdrawn and the cage was closed. Animals were observed for atypical motor responses indicative of seizure generation during and for $\sim 30$ min after infusion; behaviors quantified were jaw myoclonus (teeth chattering), lethargy or hypomobility, and wet dog shakes.

Spaced and nonspaced infusions. One of seven infusion treatments was administered: females received either a single infusion of EAA at $1.5 \mu \mathrm{M}$ (1.0 $\mu \mathrm{M}$ Asp:0.5 $\mu \mathrm{M}$ Glu plus Gly), $15 \mu \mathrm{M}$ (10 $\mu \mathrm{M}$ Asp:5 $\mu \mathrm{M}$ Glu plus Gly), or $45 \mu \mathrm{M}$ (30 $\mu \mathrm{M}$ Asp:15 $\mu \mathrm{M}$ Glu plus Gly) in $50 \mathrm{~mm}$ PBS, $\mathrm{pH}=7.4$, or three separate infusions of PBS, $1.5 \mu \mathrm{M}, 15 \mu \mathrm{M}$, or $45 \mu \mathrm{M}$ EAA given at 30 min intervals.

In separate groups of animals, blood samples were obtained for measurement of plasma PRL at the time of the nocturnal PRL surge (7:00 A.M.) and at the midpoint between the diurnal and nocturnal surges (intersurge period, 1:00 A.M.) $6 \mathrm{~d}$ after infusion, a time at which the PRL surges of P/PSP are well established (Erskine, 1995). Females given three infusions of $15 \mu \mathrm{M}$ EAA or PBS as above were bled into heparinized tubes by rapid decapitation after $\sim 15 \mathrm{~s}$ of $\mathrm{CO}_{2}$ exposure. Samples were centrifuged at $4^{\circ} \mathrm{C}$ for $20 \mathrm{~min}$, and plasma was collected and frozen at $-20^{\circ} \mathrm{C}$ until assay.

Prolonged EAA infusion. Proestrous animals received continual infusions of $15 \mu \mathrm{M}$ EAA or PBS into the MEApd at a rate of $0.02 \mu \mathrm{l} / \mathrm{min}$ over $1 \mathrm{~h}$, the total duration of the three infusions administered in the previous study. Constant infusion flow was maintained by a BAS (West Lafayette, IN) modular syringe drive and pump controller. Infusion needles were removed 4 min after the end of infusion.

Variable intervals between infusion. To determine whether the interval between EAA infusions would influence induction of P/PSP, animals were given three infusions of $15 \mu \mathrm{M}$ at 5,30 , or $60 \mathrm{~min}$ intervals, and the incidence of P/PSP was determined.

\section{Synergistic actions between mating and EAA treatments in induction of $P / P S P$}

We examined whether infusion of subthreshold EAA into the MEApd would act synergistically with intromissions to induce P/PSP. Proestrous females bearing guide cannulas directed at the MEApd as above received either a single infusion of EAA or PBS. Thirty minutes after infusion, females were placed in a mating chamber with a sexually active male and allowed to receive five nonpaced intromissions (intromission interval, $<90 \mathrm{~s}$ ). Five nonpaced intromissions is a number known to be insufficient for induction of P/PSP (Adler, 1969; Gilman et al., 1979; Erskine et al., 1989).

\section{$P R L$ radioimmunoassay}

Methods for radioimmunoassay of PRL were as reported previously (Polston et al., 1998) using rat antibody (anti-r-PRL-S-9) and standards (RP-3) provided by the National Institute of Diabetes and Digestive and Kidney Diseases (Bethesda, MD; courtesy of Dr. A. F. Parlow). Briefly, plasma was diluted in $1 \%$ bovine serum albumin (BSA; $1: 20)$ and incubated for $24 \mathrm{~h}$ with anti-rat-PRL antibody at a final tube concentration of 1:10,000. Radiolabeled PRL ( ${ }^{125} \mathrm{I}-\mathrm{PRL} ; 20,000 \mathrm{cpm} / 100 \mathrm{ml}$; PerkinElmer, Boston, MA) was added to all assay tubes, and incubation continued for an additional $24 \mathrm{~h}$. Antibody-bound isotope was precipitated by adding cold protein A (IgGSorb; Calbiochem, La Jolla, CA), and pellets were 
counted in a gamma counter. Raw data were analyzed using Microsoft (Redmond, WA) Excel software. All samples were analyzed in a single assay. The within-assay coefficient of variation was $5.79 \%$, and assay sensitivity was $30 \mathrm{pg}$.

\section{Perfusions and immunocytochemistry}

Brains of all animals were analyzed histologically for infusion placement sites. In blood-sampled animals, brains were fixed in formaldehyde by immersion after sampling. For all other animals, perfusion with $4 \%$ paraformaldehyde was performed intracardially under deep sodium pentobarbital anesthesia (Somlethal; $120 \mathrm{mg} / \mathrm{kg}$ ) 12-21 d after treatment, as described previously (Coopersmith et al., 1996). Brains were postfixed in $10 \%$ paraformaldehyde overnight followed by cryoprotection in a $25 \%$ sucrose-PBS solution. Coronal $40 \mu \mathrm{m}$ sections through the amygdala were cut on a cryostat (model 1800; Leica, Holliston, MA) and mounted on gel-coated slides. Sections were stained with cresyl violet using standard procedures, dehydrated, and coverslipped with Permount. Identification of infusion sites was determined for each animal by observing the location of damage produced by the tip of the infusion needle. Placements were considered correct if the entire $1 \mathrm{~mm}$ bevel of the infusion needle was within the MEApd, according to the atlas of Paxinos and Watson (1986). With the exception of the last experiment, data from misplaced cannulas are not presented.

Additional sections through the MEApd of both the NMDA- and EAA-infused females were stained for the DNA-binding neuronal nuclear protein (NeuN) (Mullen et al., 1992). Areas in which NeuN labeling was absent were considered to have been lesioned by the infusion treatment. Free-floating $40 \mu \mathrm{m}$ sections were washed with PBS and then incubated in a blocking buffer composed of $0.1 \%$ Saponin and $3 \%$ normal goat serum (NGS) in $0.1 \mathrm{~m}$ PBS for $30 \mathrm{~min}$ at room temperature. Sections were then incubated for $24 \mathrm{~h}$ at room temperature on a shaker with the monoclonal mouse anti-neuronal nuclei antibody (catalog \#MAB377; Chemicon, Temecula, CA) diluted at 1:1000 in a 0.1 M PBS solution containing $1.0 \%$ BSA and $1 \%$ NGS. Sections were then incubated in 1\% hydrogen peroxide and 3\% NGS in PBS. Visualization of NeuN immunoreactivity was performed using biotinylated goat antimouse IgG (Vector Laboratories, Burlingame, CA) diluted at 1:200 in $0.4 \%$ Triton X-100-PBS, the Elite Vectastain avidin-biotin horseradish peroxidase kit, and 3,3'-diaminobenzidine (Vector Laboratories) as the chromogen. Between incubation steps, all sections were washed in $0.1 \mathrm{M}$ PBS three times for $10 \mathrm{~min}$. Sections were mounted and coverslipped as above.

\section{Statistics}

With the exception of the final experiment as indicated below, only data obtained from animals having correct infusion placements were included in the statistical analyses. Statistical analysis for the incidence of P/PSP was performed with $\chi^{2}$ analysis using two-sided Fisher's exact probability tests. The number of days between estrus and the serum PRL measurements were analyzed using ANOVA followed by Student-NeumanKeuls post hoc tests.

\section{Results}

\section{Effects of NMDA infusion}

The percentage induction of P/PSP induced by unilateral intra-amygdalar NMDA infusions in anesthetized animals is shown in Figure 1. Females infused with the high dose of NMDA $(8.0 \mu \mathrm{g})$ exhibited a significantly higher incidence of P/PSP than PBS-infused controls (Fisher's exact probability; $p<0.02)$. At the low dose $(0.8 \mu \mathrm{g})$, a single infusion did not significantly increase the incidence of P/PSP. However, the group receiving three spaced infusions totaling $0.8 \mu \mathrm{g}$ NMDA $(3 \times 0.27 \mu \mathrm{g})$ showed a significantly higher incidence of P/PSP compared with the PBS controls $(p<0.01)$ and the nonspaced $0.8 \mu \mathrm{g}$ NMDA group $(p<0.03)$. There was no difference in the incidence of P/PSP between the groups receiving 8.0 and $3 \times 0.27 \mu \mathrm{g}$ NMDA. An overall ANOVA demonstrated a sig-

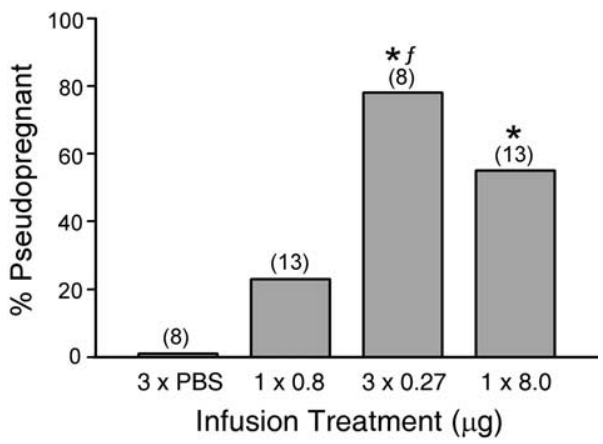

Figure 1. The effect of NMDA infusion into the MEApd on the incidence of P/PSP. Three infusions of $0.27 \mu \mathrm{g}$ of NMDA (total dose, $0.8 \mu \mathrm{g}$ ) and one infusion of $8.0 \mu \mathrm{g}$ of NMDA induced $\mathrm{P} / \mathrm{PSP}$ in a significantly greater number of animals than did infusion of PBS. The three infusions induced a significantly higher percentage of P/PSP than did a single infusion of $0.8 \mu \mathrm{g}$. The numbers in parentheses are the numbers of animals. *Significantly higher than animals receiving three infusions of PBS $(3 \times \mathrm{PBS} ; p \leq 0.01-0.02)$. ${ }^{f}$ Significantly higher than the $1 \times 0.8 \mu \mathrm{g}$ group $(p \leq 0.03)$.

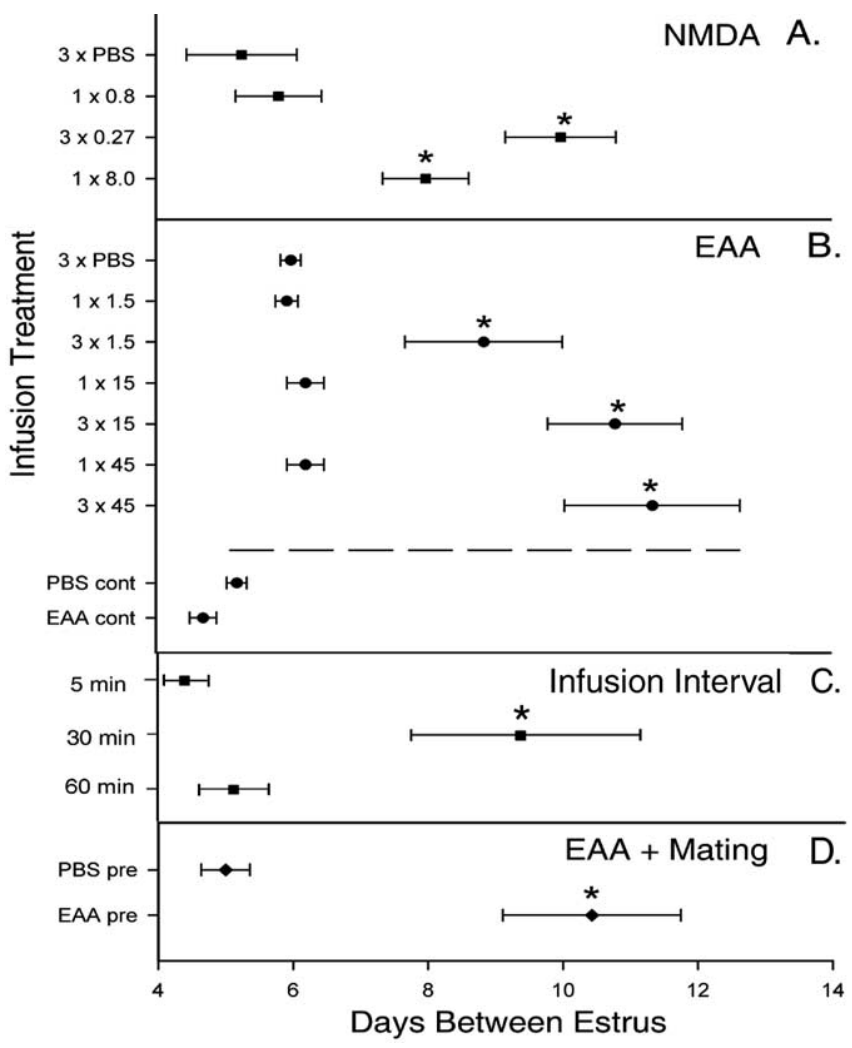

Figure 2. The mean number of days between vaginal estrus obtained by vaginal smears for NMDA-infused $(\boldsymbol{A})$ and EAA-infused $(\boldsymbol{B})$ animals, different infusion intervals $(\boldsymbol{C})$, and EAA plus mating $(\boldsymbol{D})$ experiments. The number of infusions and the doses are the same as in Figures 1 and 4. Continuous infusion (cont) occurred over $1 \mathrm{~h}$, and pretreatment infusion (pre) occurred as indicated in the text before mating stimulation. Horizontal bars are SEM. *Significantly longer periods of diestrus smears compared with PBS controls in each experiment ( $p \leq 0.04-0.001$; see Results).

nificant difference between groups in the number of days between estrus $\left(F_{(3,42)}=6.31 ; p<0.001\right)$ (Fig. $\left.2 A\right)$. Both the nonspaced $(8.0 \mu \mathrm{g})$ and the spaced $(3 \times 0.27 \mu \mathrm{g})$ treatments induced a significant increase in the number of days between estrus compared with the other two treatments $(p<0.005)$. The single $0.8 \mu \mathrm{g}$ NMDA infusion did not alter estrous cycle length. 


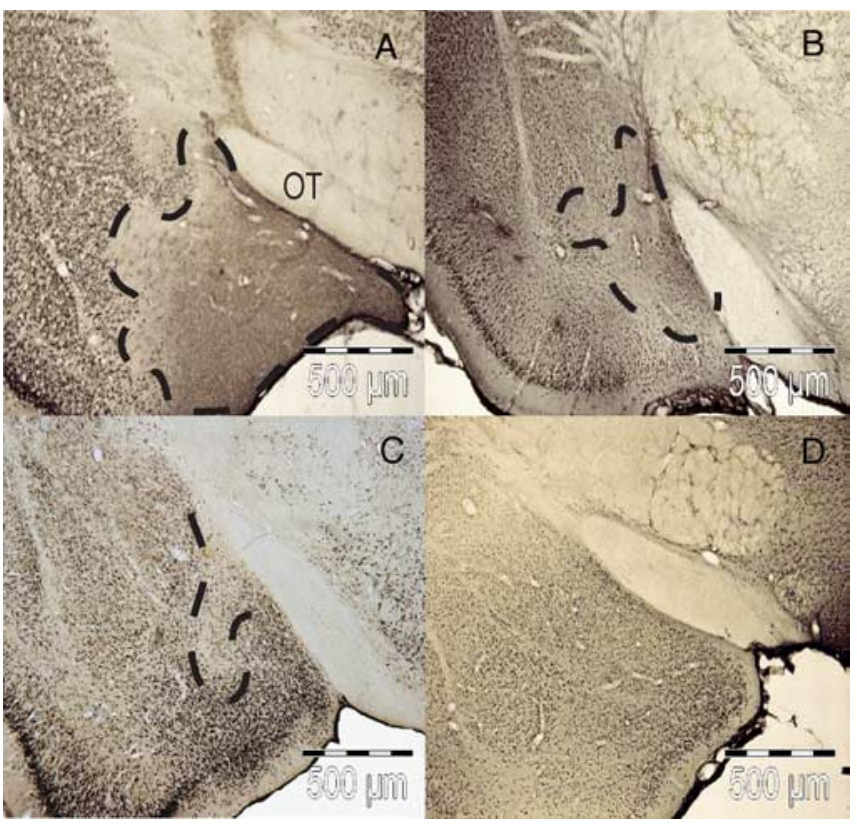

Figure 3. Photomicrographs showing staining of the nuclear neuronal protein $\mathrm{NeuN}$ in the MEApd 12-21 d after NMDA infusion. The extent of excitotoxic lesions produced by $1 \times 8.0 \mu \mathrm{g}$ of NMDA $(A), 1 \times 0.8 \mu \mathrm{g}$ of NMDA $(B), 3 \times 0.266 \mu \mathrm{g}$ of NMDA $(\boldsymbol{C})$, and $3 \times \operatorname{PBS}(\boldsymbol{D})$ is outlined with dashed lines. OT, Optic tract.

Coronal sections of the MEApd from brains taken 12-21 d after infusion and stained for $\mathrm{NeuN}$ are shown in Figure 3. The shape and extent of the excitotoxic lesions observed varied from animal to animal, but a significant loss of neurons was seen within the MEApd as well as within small, inconsistent areas extending beyond the borders of the MEApd. Single infusion of the high (Fig. 3A) and low (Fig. 3B) NMDA doses resulted in clear decreases in NeuN staining with the extent of the lesion being greater after $8.0 \mu \mathrm{g}$ than after $0.8 \mu \mathrm{g}$ infusions. In contrast, the area of reduced $\mathrm{NeuN}$ staining was somewhat less in the spaced group (Fig. $3 C$ ) than in the other treated groups. No lesion was observed in the controls given PBS infusions (Fig. 3D).

\section{Effects of EAA infusion}

As with NMDA, repeated infusions of EAA into the MEApd resulted in prolonged ovarian acyclicity characteristic of P/PSP. None of the animals that were given single infusions across a range of doses between 1.5 and $45 \mu \mathrm{M}$ became P/PSP. As seen in Figure 4, females that received three infusions of EAA at concentrations of $1.5,15$, or $45 \mu \mathrm{M}$ exhibited significantly higher incidences of P/PSP $(67,84$, and $84 \%$, respectively) compared with those receiving a single EAA infusion at the same concentrations or three PBS infusions $(p<0.02)$. Two specific comparisons are instructive. Three infusions of $1.5 \mu \mathrm{M}$ induced P/PSP in $67 \%$ of the animals, a proportion significantly higher than that produced by a single infusion of $15 \mu \mathrm{M}$, a 10 -fold higher dose $(p<0.02)$. In addition, three infusions of $15 \mu \mathrm{M}$ resulted in a high incidence of $\mathrm{P} / \mathrm{PSP}$, whereas a single infusion of three times that dose $(45 \mu \mathrm{M})$ did not induce P/PSP in any animal $(p<0.02)$. There was a significant effect of infusion treatment on the days between estrus $\left(F_{(8,45)}=9.64 ; p<0.001\right)$ (Fig. $\left.2 B\right)$. The mean numbers of days between estrus were significantly higher in the groups receiving three infusions of 15 or $45 \mu \mathrm{M}$ than in groups receiving single infusions $(p<0.001)$, but the three infusions of $1.5 \mu \mathrm{M}$ did not lengthen the period of diestrus significantly.

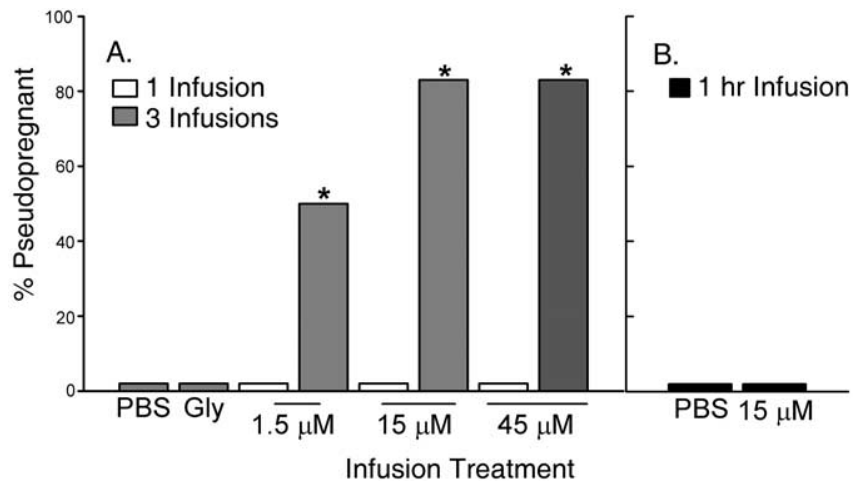

Figure 4. The effect of EAA infusion into the MEApd on the incidence of P/PSP. Three infusions of EAA were significantly more effective in inducing P/PSP at all doses tested than were single infusions $(\boldsymbol{A})$. Three infusions of PBS or Gly alone did not induce P/PSP in any animal. Animals receiving continuous infusions of EAA $(15 \mu \mathrm{m})$ or PBS over $1 \mathrm{~h}$ also did not show P/PSP $(\boldsymbol{B})$. The number of animals per group receiving one or three infusions was six. Gly-infused and continuously infused groups consisted of five animals. *Significantly higher than groups receiving a single infusion at the same concentration ( $p \leq 0.02$ ).

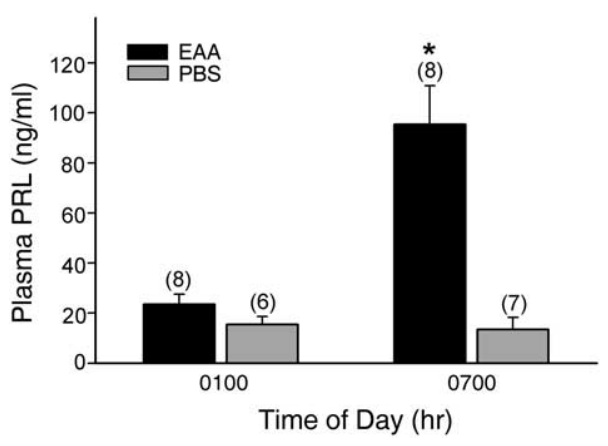

Figure 5. Plasma PRL concentrations (mean \pm SEM) in samples obtained from animals given three infusions of EAA (15 $\mu \mathrm{m})$ or PBS $6 \mathrm{~d}$ previously. PRL levels were low during the intersurge period (1:00 A.M.), whereas only EAA-infused animals showed increased PRL levels at the time of the nocturnal surge (7:00 A.M.). The numbers in parentheses are the numbers of animals per group. *Significantly higher than all other times and treatments ( $p \leq 0.001)$.

In contrast to the effects of multiple infusions, continuous infusion of EAA at a dose of $15 \mu \mathrm{M}$ over $1 \mathrm{~h}$, the time span over which the three spaced infusions were performed, did not induce $\mathrm{P} / \mathrm{PSP}$ in any animal (Figs. $2 \mathrm{~B}, 4 \mathrm{~B}$ ). The mean number of days between estrus in the continuously infused EAA animals was not different from the number exhibited by cycling continuously infused PBS females (Fig. 2B). Thus, these data suggest that the effectiveness of the three infusions was not solely the result of longer tissue exposure to EAA than that produced by the single infusions.

Plasma PRL concentrations measured at the time of the nocturnal surge and the intersurge interval in both P/PSP and non$\mathrm{P} / \mathrm{PSP}$ animals are presented in Figure 5. A two-way ANOVA revealed significant effects of both infusion treatment $\left(F_{(1,23)}=\right.$ 52.89; $p<0.001)$ and sample time $\left(F_{(1,23)}=36.84 ; p<0.001\right)$ and an interaction between infusion treatment and sample time $\left(F_{(1,23)}=39.76 ; p<0.001\right)$. The presence of high concentrations of PRL in the EAA-treated animals at the time of the nocturnal surge confirmed that the vaginal acyclicity induced by three EAA infusions was accompanied by the surge secretion of PRL characteristic of P/PSP. PRL concentrations in EAA-treated females at 7:00 A.M. were significantly higher than those observed in the PBS-treated controls at that time $(p<0.001)$ and were signifi- 


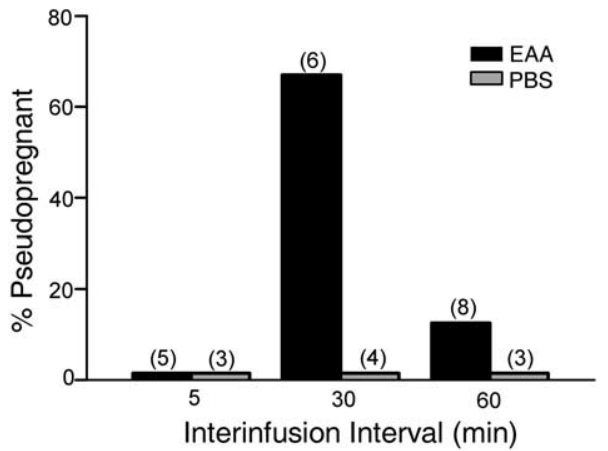

Figure 6. Effect of differing intervals between the three infusions of EAA on the incidence of P/PSP. A greater number of animals receiving three infusions of EAA (15 $\mu \mathrm{M})$ at 30 min intervals showed P/PSP than did animals receiving three infusions at 5 min intervals ( ${ }^{*} p \leq 0.04$ ). The interinfusion time did not alter the lack of response to PBS.

cantly higher than levels measured in both groups at 1:00 A.M. $(p<0.001)$.

Neither single, triple, nor continuous infusions of EAA in conscious rats produced detectable decreases in the number of neurons surrounding the needle tract $12-21 \mathrm{~d}$ after infusion in the MEApd of infused animals (data not shown).

\section{Behavioral responses to infusion}

The infusion treatments resulted in various behavioral responses, including jaw myoclonus, ptosis of the eyes, wet dog shakes, and initial hypoactivity followed by hyperactivity. These behaviors occurred within the first minute of infusions and persisted for a maximum of $10 \mathrm{~min}$ from onset. Furthermore, in animals receiving multiple stimulations, the majority of the responses occurred within the first infusion, rarely developed during the second, and never during the third infusion. These behavioral patterns were observed in all experimental groups, including Gly-only and PBS-infused control animals. The occurrence of these behaviors in all treatment groups coupled with the absence of more severe seizure behaviors suggests that the EAA infusion paradigm did not result in ectopic spread of neural activity.

\section{Effects of variation in the time interval between infusions}

Differential effects of the interval between three EAA infusions are shown in Figure 6. There was a statistically significant overall effect of infusion interval $\left(F_{(1,23)}=4.08 ; p<0.008\right)$. Infusion of $15 \mu \mathrm{M}$ EAA at 30 min intervals induced P/PSP in a significantly higher number of females $(67 \%)$ than did the EAA infusions at 5 min intervals $(0 \% ; p<0.04$; one-tailed test $)$. Because of the small number of animals, comparisons between the $30 \mathrm{~min}$ interval EAA-infused group and the $30 \mathrm{~min}$ interval PBS $(p<$ $0.07)$ and 60 min interval EAA $(p<0.06)$ groups approached but did not reach statistical significance. However, as seen in Figure $2 C$, the mean number of days between estrus in the 30 min EAA group was significantly longer than all other treatments including the 5 min interval and the $60 \mathrm{~min}$ interval EAA groups $(p<0.04)$.

\section{Synergism between mating stimulation and chemical stimulation of the MEApd}

To address the question of whether EAA release within the MEApd in response to individual intromissions is involved in initiation of P/PSP, we examined whether EAA would be additive with mating stimulation for attainment of the threshold for

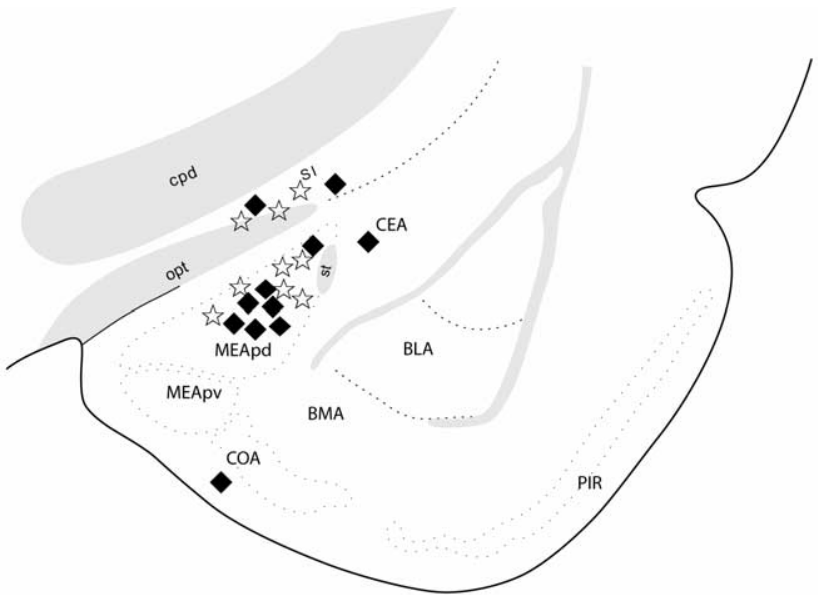

Figure 7. Location of single infusions of EAA (stars) and PBS (filled diamonds) followed 30 min later by subthreshold mating stimulation. Placements outside the delineated MEApd were considered misplacements (AP level shown, -2.45 from bregma). BLA, Basolateral amygdala; BMA, basomedial amygdala; CEA, central amygdala; $C O A$, cortical amygdala; cpd, cerebral peduncle; MEApv, posteroventral medial amygdala; opt, optic tract; PIR, piriform cortex; SI, substantia inominata; st, stria terminalis.

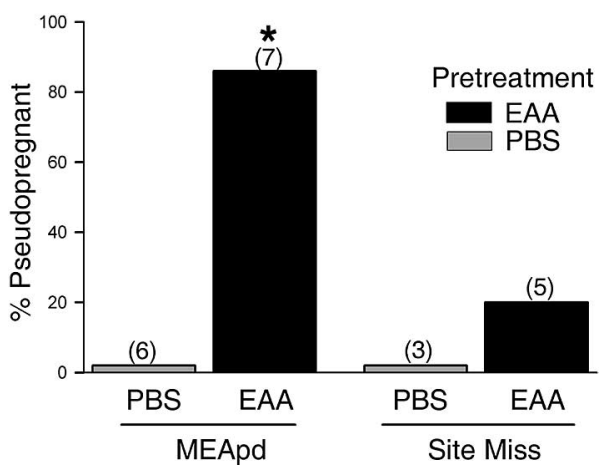

Figure 8. Percentage of animals showing P/PSP after a subthreshold infusion of EAA followed by subthreshold mating stimulation. Animals pretreated with a single MEApd infusion of $15 \mu \mathrm{m}$ EAA were significantly more likely to become P/PSP after mating than animals pretreated with PBS. Pretreatment with EAA in sites that missed the MEApd produced no significant increase in P/PSP. The numbers in parentheses are the numbers of animals per group. * Significantly higher than animals receiving PBS in the MEApd ( $p \leq 0.005)$.

$\mathrm{P} / \mathrm{PSP}$ initiation. We treated females with a single intraamygdalar infusion of an amount of EAA shown to be subthreshold for induction of P/PSP $(15 \mu \mathrm{M})$ followed by mating stimulation (five intromissions) also known to be subthreshold for $\mathrm{P} / \mathrm{PSP}$. The location of the infusions is shown in Figure 7. EAA did not influence the expression of sexual behavior $30 \mathrm{~min}$ after infusion. All animals in both the EAA and PBS infusion groups showed lordosis responses to every mount or intromission received.

Figure 8 shows the effects of five intromissions on the incidence of P/PSP in animals that had received either a single EAA or PBS infusion $30 \mathrm{~min}$ before the onset of the mating test. Combination of EAA infusion with five intromissions induced P/PSP in $86 \%$ of the animals tested. This percentage was comparable with that seen previously after three spaced infusions of NMDA and EAA and was significantly higher than that exhibited by PBSpretreated animals $(p<0.005)$. In the latter group, five intromissions did not induce P/PSP in any animal. Data from animals with infusions that were not correctly placed within the MEApd were used as negative controls to determine the importance of 
site specificity; incorrect placements were within the cortical or basolateral amygdala or above the optic tract. As shown in Figure 8, EAA infusions outside the MEApd induced a small P/PSP response to the mating stimulation (one of five), which was not different from either group receiving correct (zero of six) or incorrect (zero of three) PBS infusion placements. The mean number of days between estrus (Fig. 2D) was significantly greater in the animals receiving EAA within the MEApd before five intromissions than in animals receiving $\operatorname{PBS}\left(F_{(1,11)}=95.21 ; p<\right.$ $0.001)$.

\section{Discussion}

The present results indicate that properly timed, repetitive activation of glutamate receptors within the MEApd initiates longterm alterations in brain function that are responsible for the neuroendocrine changes of P/PSP. Direct application of NMDA or EAA into the MEApd initiated persistent ovarian acyclicity and PRL secretory responses (Polston et al., 2001; present results), events normally initiated by an estrous female's receipt of VCS during mating (Erskine, 1995). Furthermore, direct chemical stimulation of the MEApd is more likely to induce a neuroendocrine response when the stimulation is temporally spaced. Three temporally spaced infusions of either NMDA or EAA administered at low, individually subthreshold doses were more effective than single infusions in initiating P/PSP. Finally, when subthreshold mating stimulation and subthreshold chemical stimulation of the MEApd were combined, a strong synergistic interaction occurred, which resulted in P/PSP. These findings strengthen considerably the hypothesis that glutamate released repetitively in the MEApd in response to intromissions during mating induces P/PSP.

In the freely behaving animal, the successful induction of $\mathrm{P} / \mathrm{PSP}$ is a function of both the number (Adler, 1969; Terkel and Sawyer, 1978) and temporal patterning or rate (Erskine et al., 1989) of VCS received by the estrous female. Therefore, single, individually subthreshold neural changes must be cumulative for attainment of the threshold for P/PSP. This accumulation of repeated intromissive stimuli appears to be dependent on repetitive activation of NMDA receptors within the MEApd. Whereas single infusions of EAA were ineffective, the equivalent dosage divided into three separate infusions spaced $30 \mathrm{~min}$ apart induced a $\mathrm{P} / \mathrm{PSP}$ response. This result shows that chemically induced mnemonic acquisition is sensitive to repeated glutamate receptor activation within the MEApd. An exception would appear to be the $\mathrm{P} / \mathrm{PSP}$ response observed in the group receiving the single high dose of NMDA ( $8 \mu \mathrm{g}$ or $136 \mathrm{mmol}$ ), which replicated previous findings (Numan et al., 1993; Polston et al., 2001). However, the large lesion footprint produced by this infusion and the relatively low affinity of NMDA for the Glu transporter system (Balcar and Johnston, 1972; Davies and Johnston, 1976; Skerritt and Johnston, 1981) make it possible that this high dose stimulated a greater number of MEApd neurons and/or stimulated receptors for a longer duration than did the single EAA or low-dose NMDA infusions.

The induction of P/PSP also was modulated by the rate of infusions. We selected the $30 \mathrm{~min}$ interval as likely to provide discrete individual signals, given that the clearance rate of Glu has been reported to be between 15 and $100 \mu \mathrm{M}$ per $60 \mathrm{~min}$ in cultured astrocytes (Abe and Saito, 2000; Abe et al., 2000; Abe and Misawa, 2003). Although the $30 \mathrm{~min}$ interval is longer than the known optimal behavioral interval ( $\sim 3-5 \mathrm{~min})$ between intromissions (Edmonds et al., 1972), it has been shown that summation of intromissive stimuli occurs when enforced interintromis- sion intervals are as long as $30 \mathrm{~min}$ (Edmonds et al., 1972). In fact, three EAA infusions at $15 \mu \mathrm{M}$ applied at $30 \mathrm{~min}$, but not 5 or 60 min, intervals elicited a P/PSP response. Inherent differences between synaptic release after VCS and bolus experimental administration of neurotransmitter may explain why the shorter interinfusion interval failed to induce P/PSP. The lack of responsiveness of postsynaptic neurons to stimuli administered continuously or at closely spaced intervals may have resulted from desensitization of glutamate receptors in the MEApd. It has been shown that desensitization of the NMDA receptor occurs within the striatum when repeated local injections of NMDA $(100 \mu \mathrm{M})$ are given $80-90 \mathrm{~s}$ apart but not at $30 \mathrm{~min}$ intervals (Crespi and Rossetti, 2004). Also, a large acidic amino acid-induced depolarization of medial hypothalamic neurons was shown to decay with repetitive application (Arnauld et al., 1983). Reduced c-fos expression in response to closely spaced, as opposed to more widely spaced, intromissions has also been observed within the MEApd (Erskine and Hanrahan, 1997).

Excitation of MEApd neurons by NMDA rather than excitotoxicity (Dusart et al., 1991; Marty et al., 1991) is likely responsible for P/PSP induction in the first experiment. Loss of function of glutamate-sensitive cells would be predicted to cause effects similar to those of glutamate antagonists, yet, as indicated above, AP-5 infusion before mating prevented rather than stimulated mating-induced P/PSP. In addition, treatment with a lower concentration of NMDA consistently resulted in lesions of MEApd neurons but did not induce P/PSP, whereas EAA infusion treatments induced P/PSP but resulted in no observable neuronal damage. The mixture of Asp and Glu we used was adapted from previous research (Mori and Wada, 1987; Croucher and Bradford, 1989) that demonstrated that repeated spaced injections of this mixture into the amygdala induced progressive seizure development with minimal histological damage. Both NMDA and non-NMDA receptors are activated by L-glutamate, whereas L-aspartate appears to activate NMDA receptors exclusively (Kleckner and Dingledine, 1988). However, we did not observe behavioral responses to repetitive infusions indicative of kindling of the MEApd. The addition of glycine, which potentiates NMDA receptor activation at submicromolar levels (Johnson and Ascher, 1987) and is an essential coagonist at NMDA receptors (Kleckner and Dingledine, 1988), was held at a low dose, which of itself had no effect on P/PSP.

The MEApd appears to be particularly important to the initial transduction of VCS and to acquisition of the mnemonic for $\mathrm{P} / \mathrm{PSP}$. This area shows greatly increased levels of c-fos expression after both natural and artificial mechanosensory VCS and is the single area out of several mating-responsive brain sites in which c-fos expression changes in response to subtle changes in the number (Polston and Erskine, 1995) and rate (Erskine and Hanrahan, 1997) of intromissions received. Furthermore, bilateral transection of the pelvic nerve, a treatment that completely eliminates P/PSP (Carlson and DeFeo, 1965), dramatically reduced the number of Fos-immunoreactive neurons in this region after copulation (Rowe and Erskine, 1993). Lidocaine infusions into the MEApd but not the medial preoptic area before mating blocked P/PSP (Coopersmith et al., 1996). We do not know at present what cellular or physiological changes occur after EAA stimulation within the MEApd or at downstream sites that result in P/PSP. Infusion of AP- 5 before mating blocked mating-induced c-fos expression in the bed nucleus of the stria terminalis and the ventromedial nucleus of the hypothalamus (Polston et al., 2001), suggesting transfer of 
information from the MEApd to these areas. Regardless, it seems likely that the MEApd is involved in the initial transduction of VCS, rapidly processing and accumulating sensory information over the course of the mating session and then relaying this information to other areas of the limbic system where long term storage and the subsequent expression of the PRL surges are controlled.

Strong evidence for a common mode of action (i.e., via glutamate receptor activation) of VCS-induced and chemically induced P/PSP is the synergism we observed between these two modes of stimulation. Previous mating experiments have determined that low numbers (less than five) of nonpaced intromissions are subthreshold for P/PSP induction (Adler, 1969; Gilman et al., 1979; Erskine et al., 1989). Likewise, none of the single infusion doses of EAA in the current studies proved able to effect estrous cyclicity and so must be considered subthreshold stimuli. It is not known whether the five nonpaced intromissions stimulate EAA release within the MEApd or, if so, how much is released. Because spacing of EAA stimuli is critical for initiation of $\mathrm{P} / \mathrm{PSP}$, it is possible that the single $30 \mathrm{~min}$ interval between the infusion and mating had a greater influence on P/PSP initiation than did the amount of EAA released endogenously in response to the five nonpaced intromissions. The observation that pretreatment with a single subthreshold infusion of EAA significantly enhanced the ability of subthreshold mating treatment to induce P/PSP revealed a synergistic effect between EAA and intromissions in the MEApd. These findings provide strong evidence for a common mode of action via glutamate receptor activation. Thus, we show that the patterning of VCS during female sexual behavior is encoded faithfully within the MEApd by patterned activation of the NMDA receptor. This activation is a critical step in a series of neurobiological changes by which sexual behavior initiates the expression of the long-term neuroendocrine changes that are required for pregnancy.

These data raise interesting questions about how repeated EAA or genitosensory stimuli induce experience-dependent changes in brain function in rats. We have yet to understand the neurophysiological or molecular events that are induced within the MEApd by either EAA infusion or intromissive stimulation from males. If, as suggested by Davis (2004), mechanisms for certain types of memory are shared by different classes of animals, it is appropriate to examine the similarities between mating-induced P/PSP and forms of nonassociative learning in other species that occur in response to repeated stimuli. Like the present results, studies in Drosophila (Tully et al., 1994), honeybees (Menzel et al., 2001), Aplysia (Sutton et al., 2002), and mice (Kogan et al., 1997; Woo et al., 2003) have demonstrated that spaced rather than massed stimuli enhance memory formation. This aspect of the mating-induced P/PSP response system may be particularly useful for identifying common mechanisms across species that control learning and memory.

\section{References}

Abe K, Misawa M (2003) Amyloid beta protein enhances the clearance of extracellular L-glutamate by cultured rat cortical astrocytes. Neurosci Res 45:25-31.

Abe K, Saito H (2000) Involvement of $\mathrm{Na}+-\mathrm{K}+$ pump in L-glutamate clearance by cultured rat cortical astrocytes. Biol Pharm Bull 23:1051-1054.

Abe K, Abe Y, Saito H (2000) Evaluation of L-glutamate clearance capacity of cultured rat cortical astrocytes. Biol Pharm Bull 23:204-207.
Adler NT (1969) Effects of the male's copulatory behavior on successful pregnancy of the female rat. J Comp Physiol Psychol 69:613-622.

Arnauld E, Layton BS, Padjen AL, Renaud LP (1983) Actions of acidic amino acids on the excitability of medial hypothalamic neurons in the rat. Neuroendocrinology 37:184-192.

Balcar VJ, Johnston GAR (1972) The structural specificity of the high affinity uptake of L-glutamate and L-aspartate by rat brain slices. J Neurochem 19:2657-2666.

Carlson RR, DeFeo VJ (1965) Role of the pelvic nerve vs. the abdominal sympathetic nerves in the reproductive function of the female rat. Endocrinology 77:1014-1022.

Coopersmith C, Gans SE, Rowe DW, Erskine MS (1996) Infusions of lidocaine into the amygdala, but not the preoptic area, block pseudopregnancy in the rat. J Neuroendocrinol 8:259-266.

Crespi F, Rossetti ZL (2004) Pulse of nitric oxide release in response to activation of $N$-methyl-D-aspartate receptors in the rat striatum: rapid desensitization, inhibition by receptor antagonists, and potentiation by glycine. J Pharmacol Exp Ther 309:462-468.

Croucher MJ, Bradford HF (1989) Kindling of full limbic seizures by repeated microinjections of excitatory amino acids into the rat amygdala. Brain Res 50:58-65.

Davies LP, Johnston GAR (1976) Uptake and release of D- and L-aspartate by rat brain slices. J Neurochem 26:1007-1114.

Davis RL (2004) Olfactory learning. Neuron 44:31-48.

Dusart I, Marty S, Peschanski M (1991) Glial changes following an excitotoxic lesion in the CNS-II. Astrocytes. Neuroscience 45:541-549.

Edmonds S, Zoloth SR, Adler NT (1972) Storage of copulatory stimulation in the female rat. Physiol Behav 8:161-164.

Erskine MS (1995) Prolactin release after mating and genitosensory stimulation in females. Endocr Rev 16:508-528.

Erskine MS, Hanrahan SB (1997) Effects of paced mating on c-fos gene expression in the female rat brain. J Neuroendocrinol 9:903-912.

Erskine MS, Kornberg E, Cherry JA (1989) Paced copulation in rats: effects of intromission frequency and duration on luteal activation and estrus length. Physiol Behav 45:33-39.

Gilman DP, Mercer LF, Hitt JC (1979) Influence of female copulatory behavior on the induction of pseudopregnancy in the female rat. Physiol Behav 22:675-678.

Gunnet JW, Freeman ME (1983) The mating-induced release of prolactin: a unique neuroendocrine response. Endocr Rev 4:44-61.

Johnson JW, Ascher P (1987) Glycine potentiates the NMDA response in cultured mouse brain neurons. Nature 325:529-531.

Kleckner NW, Dingledine R (1988) Requirement for glycine in activation of NMDA-receptors expressed in Xenopus oocytes. Science 241:835-837.

Kogan JH, Frankland PW, Blendy JA, Coblentz J, Marowitz Z, Schutz G, Silva AJ (1997) Spaced training induces normal long-term memory in CREB mutant mice. Curr Biol 7:1-11.

Marty S, Dusart I, Peschanski M (1991) Glial changes following an excitotoxic lesion in the CNS-I. Microglia/macrophages. Neuroscience 45:529-539.

Menzel R, Manz G, Menzel R, Greggers U (2001) Massed and paced learning in honeybees: the role of CS, US, the intertrial interval, and the test interval. Learn Mem 8:198-208.

Mori N, Wada JA (1987) Bidirectional transfer between kindling induced by excitatory amino acids and electrical stimulation. Brain Res 425:45-48.

Mullen RJ, Buck CR, Smith AM (1992) NeuN, a neuronal specific nuclear protein in vertebrates. Development 116:201-211.

Numan M, Numan MJ, English JB (1993) Excitotoxic amino acid injections into the medial amygdala facilitate maternal behavior in virgin female rats. Horm Behav 27:56-81.

Paxinos G, Watson C (1986) The rat brain in stereotaxic coordinates. San Diego: Academic.

Polston EK, Erskine MS (1995) Patterns of induction of the immediateearly genes c-fos and egr- 1 in the female rat brain following differential amounts of mating stimulation. Neuroendocrinology 62:370-384.

Polston EK, Centorino KM, Erskine MS (1998) Diurnal fluctuations in mating-induced oxytocinergic activity within the paraventricular and supraoptic nuclei do not influence prolactin secretion. Endocrinology 139:4849-4859.

Polston EK, Heitz M, Barnes W, Cardamone K, Erskine MS (2001) NMDAmediated activation of the medial amygdala initiates a downstream neu- 
roendocrine memory responsible for pseudopregnancy in the female rat. J Neurosci 21:4104-4110.

Rowe DW, Erskine MS (1993) c-Fos proto-oncogene activity induced by mating in the preoptic area, hypothalamus and amygdala in the female rat: role of afferent input via the pelvic nerve. Brain Res 621:25-34.

Skerritt JH, Johnston GAR (1981) Uptake and release of N-methyl-Daspartate by rat brain slices. J Neurochem 36:881-885.

Smith MS, Neill JD (1976) Termination at midpregnancy of the two daily surges of plasma prolactin initiated by mating in the rat. Endocrinology 98:696-701.

Smith MS, McLean BK, Neill JD (1976) Prolactin: the initial luteotropic stimulus of pseudopregnancy in the rat. Endocrinology 98:1370-1377.

Sutton MA, Ide J, Masters SE, Carew TJ (2002) Interaction between amount and pattern of training in the induction of intermediated- and long-term memory for sensitization in Aplysia. Learn Mem 9:29-40.

Terkel J, Sawyer CH (1978) Male copulatory behavior triggers nightly prolactin surges resulting in successful pregnancy in rats. Horm Behav 11:304-309.

Tetel MJ, Getzinger MJ, Blaustein JD (1993) Fos expression in the rat brain following vaginal-cervical stimulation by mating and manual probing. J Neuroendocrinol 5:397-404.

Tully L, Preat T, Boynton SC, Del Vecchio M (1994) Genetic dissection of consolidated memory in Drosophila. Cell 79:35-47.

Woo NH, Duffy SN, Abel T, Nguyen PV (2003) Temporal spacing of synaptic stimulation critically modulates the dependence of LTP on cyclic AMP-dependent protein kinase. Hippocampus 13:293-300. 